\title{
Sculpting the vacuum in a photonic band gap micro-chip
}

\author{
Sajeev John*, Rongzhou Wang \\ Department of Physics, University of Toronto, 60 St. George Street, Toronto, Ont., Canada M5S 1A7
}

Received 22 June 2004; received in revised form 12 July 2004; accepted 12 July 2004

Available online 3 August 2004

\begin{abstract}
We describe the engineering of the electromagnetic vacuum in a 2D-3D photonic bandgap (PBG) hetero-structure. This facilitates the development of novel active devices and the observation of novel quantum electrodynamic phenomena. We consider a specific architecture suitable as an all-optical micro-transistor capable of novel ultra-fast response with low switching power requirements. This relies on a unique collective atomic switching and population inversion achieved by coherent resonant pumping in a suitably engineered vacuum. Specific waveguide architectures within the 3D PBG micro-chip provide local density-of-states (LDOS) peaks near their cutoff frequency. These provide "building blocks" for electromagnetic vacuum engineering without recourse to conventional high Q-factor micro-cavities. For the all-optical micro-transistor, a fork shape LDOS within the micro-chip is desirable, using trimodal waveguide architecture. We delineate the functional robustness of these architectures to disorder caused by manufacturing errors within the PBG micro-chip.

(C) 2004 Elsevier B.V. All rights reserved.
\end{abstract}

PACS: 42.70.Qs; 42.55.Tv; 42.50.-p

Keywords: Electromagnetic vacuum; Photonic bandgap; Micro-chip

\section{Introduction}

Photonic bandgap (PBG) materials [1,2] are artificial periodic dielectrics that enable the engineering of the most fundamental properties of electromagnetic waves. These properties include extraordinary optical refraction [3], light flow without diffraction [4], frequency selective control of spontaneous emission [5], an engineered blackbody radiation spectrum [6], and

\footnotetext{
* Corresponding author. Tel.: +1 416978 3459; fax: +1 4169782537.

E-mail address: john@physics.utoronto.ca (S. John).
}

other fundamental aspects of radiation-matter interactions [7]. One of the most unique properties of PBG materials is their ability to localize light [1]. This novel confinement mechanism provides a basis for practical applications such as guiding of light in the air-core of structured fibers [8] and the micro-manipulation of diffractionless light through air-waveguides in a PBG micro-chip [4].

In this paper, we describe ways in which diffractionless flow of light in a 3D PBG micro-chip can be combined with electromagnetic density-of-states engineering. This facilitates the integration of novel passive optical micro-circuit paths with microscopic 
active devices into an all-optical micro-chip. Unlike electronic micro-circuits limited to a single "channel" of information transfer and processing, optical microcircuitry in a 2D-3D PBG heterostructure exhibits a bandwidth (for single-mode waveguiding) of roughly $185 \mathrm{~nm}$ at a center wavelength of $1.5 \mu \mathrm{m}[4,9]$. This facilitates hundreds of channels of data to be processed in parallel from a wavelength division multiplexed (WDM) network. In this context, it is possible to engineer the electromagnetic vacuum in order to controllably alter atomic response to a radiation field. For example, the modifications of the decay lifetime of dye molecules in PCs without a PBG have been observed in experiments [10]. When the atomic transition frequency is inside a PBG, a photon-atom bound state is formed, leading to a total prohibition of the decay of the atom by single photon emission [11]. Alternatively, if the transition frequency of the atom is near a LDOS discontinuity, a collection of such atoms can be coherently driven by a pump laser into a steady state population-inverted configuration [12]. This is impossible in an ordinary vacuum, where atomic response is governed by the Einstein rate equations [13].

$N$ atoms collective switching [14] is suitable for an all-optical micro-transistor because it can greatly improve the performance of the switching behavior [15]. Near the threshold value, the modulation intensity of the pump laser required to "switch" the atomic population decreases linearly as $1 / N$. Collective atomic response also makes the switching behavior robust to dephasing interactions (which would eventually smear out the single-atom population inversion). In comparison to other all-optical transistor proposals [16-19], collective atomic switching in a PBG is not constrained by the inverse relationship between switching time and switching threshold[14]. This undesirable trade-off is usually associated with any device involving a high Q-factor cavity such as a nonlinear Fabry-Perot interferometer [16,17]

In practice, quantum dots (instead of the two-level atoms) may be more suitable for an optical transistor device because they can easily be grown and precisely positioned on a structured semiconductor surface [20]. Usually, an ensemble of quantum dots will exhibit an inhomogeneously broadened atomic resonance frequency. In this case, a fork shaped LDOS is required to realize atomic population inversion [21]. Atoms detuned from the prescribed LDOS cannot couple to either the external fields or the electromagnetic (vacuum) reservoir and as a result the deleterious part of the atomic distribution is quenched.

The engineering of the electromagnetic LDOS can also be used to fabricate a one-atom micro-laser [22]. Here, one or more quantum dots are coupled strongly to both a high $\mathrm{Q}$ micro-cavity and the engineered electromagnetic reservoir of a photonic crystal microchip. By suitably engineering this electromagnetic LDOS, it is possible to controllably alter the quantum statistics of photons emitted by the Q-dot into the micro-cavity. The required LDOS here is a combination of a multi-waveguide mode and a cavity mode. The multi-waveguide mode provides a channel for the pumping laser beam and a big jump of LDOS for atomic population inversion. The laser emits through the cavity mode. We use waveguides as "building blocks" to design the electromagnetic vacuum. Near the cut-off frequency $\left(\omega_{\mathrm{c}}\right)$, the group velocity of light approaches zero, leading to a square-root divergence in the DOS in an infinite sample. This divergence of the DOS is interrupted by either the finite size of the sample or the finite length of the engineered waveguide section, leading to a LDOS peak with a finite height.

The paper is organized as follows. In Section 2, we briefly review the 2D-3D PBG heterostructures. The mechanism of atomic population inversion is briefly reviewed in Section 3. In Section 4, the properties of the LDOS of a waveguide in a 2D-3D heterostructure is studied. Using 2D PCs, the desired LDOS for alloptical transistor is designed in Section 5. In Section 6, the robustness of the LDOS architecture to disorder is studied. Finally, a summary is presented in Section 7.

\section{Photonic bandgap micro-chips based on 2D-3D heterostructures}

When light propagates in a 2D PC membrane, attenuation of the guided optical signal may occur due to leaky modes into the third dimension. Even weak disorder can induce small angle forward scattering into the third dimension. However, a 3D PBG heterostructure [4] can prevent light from leaking into the third dimension, even in the presence of the deviation from periodicity or unintentional forms of random 
disorder on the micro-chip layer. This is a great advantage for integrated all-optical microcircuits. The cladding 3D PC can be a square spiral [23], a woodpile [24], a slanted pore [25], or an inverse opal [26], etc. A pictorial illustration is given in Fig. 1 using a woodpile as the 3D cladding material. In order to obtain singlemode, broad-bandwidth diffractionless light propagation, it is important to match the field distributions in the 2D PC slab region with the 3D PBG over all wave vectors available to the 2D micro-chip [9].

The 2D slab can be regarded as a planar defect within the 3D PBG material, that introduces defect modes into the 3D PBG. These defect modes will occupy the upper fraction of the original 3D PBG, but a complete on-chip 3D PBG is retained. The size of this on-chip bandgap decreases with the thickness of the 2D micro-chip layer, disappearing completely if the layer is thick enough. Additional point and line

(a)

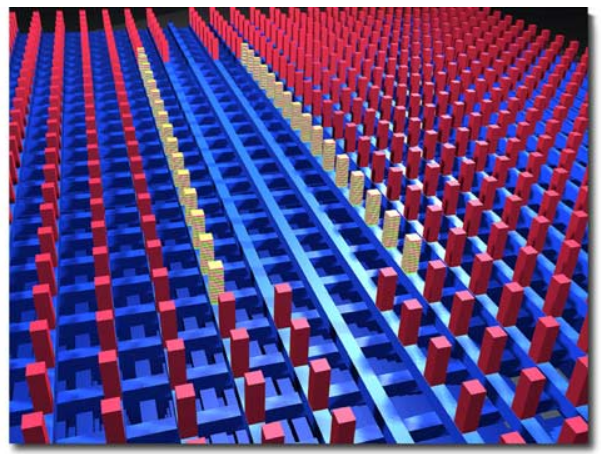

(b)

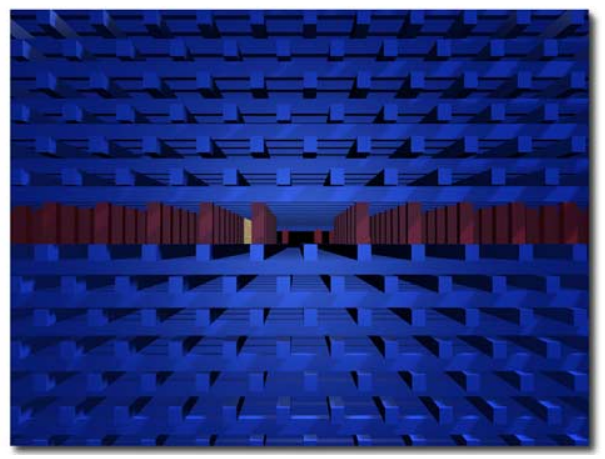

Fig. 1. The hetero-structure with woodpile structure as the upper and lower 3D PBG cladding sections. The middle part is a W3 waveguide with highlighted rods in which quantum dots (or twolevel atoms) are integrated. The ends of the W3 waveguide are connected by W1 waveguides. (a) Bird's eye view with the upper cladding section removed. (b) Side view with both upper and lower cladding sections. defects within the the 2D micro-chip layer may provide further circuit elements within the on-chip 3D PBG. Removing one line (three lines) of rods or holes in the 2D PC micro-chip layer will create a W1 (W3) waveguide. We focus on the air-waveguides consisting of a single row of missing dielectric rods in the 2D PC micro-chip. Such air-waveguides are readily engineered to be single-mode, while dielectric waveguides with a single row of missing holes tend to be multimode. Single-mode waveguide channels are more robust (exhibit lower scattering losses) to imperfections in manufacturing than their multi-mode counterparts.

In this section, we use direct diamond:1-square spirals [23,9] as the cladding materials to show the properties of the heterostructures. This kind of square spirals has already been synthesized using glancing angle deposition techniques [27]. The parameters of the optimized diamond: 1 structure are $[L, c, r]=$ $[0.70,1.35,0.20] a$, which correspond to a dielectric volume fraction of $\sim 31 \%$. This structure possesses a $3 \mathrm{D}$ PBG of $\sim 14.8 \%$, which is rather modest compared with $\sim 23 \%$ in the case of inverse diamond:5. Remarkably, the direct diamond: 1 structure is among the best to support a large waveguide bandwidth of micro-circuitry for diffractionless light flow.

Usually, a thinner 2D layer creates a large on-chip PBG. Unfortunately, in this case, the waveguide dispersion relation is so flat that it does not span the whole on-chip PBG, resulting in a small single-mode bandwidth. On the other hand, a thicker $2 \mathrm{D}$ layer provides a waveguide mode of a large frequency range, but the on-chip PBG is small. Optimization of the waveguide bandwidth requires the balance between the size of the on-chip PBG and the resulting waveguide dispersion. When the thickness of the 2D layer is $h=0.55 a$ and the radius of the rods in the 2D slab is $r=0.16 a$, the 2D-3D photonic crystal heterostructure has an optimized single-mode waveguide bandwidth of $\sim 185 \mathrm{~nm}$, as depicted in Fig. 2 .

\section{Atomic population inversion in an engineered vacuum}

An intriguing application of LDOS engineering is related to the possibility of coherently switching a collection of two-level system into a population- 


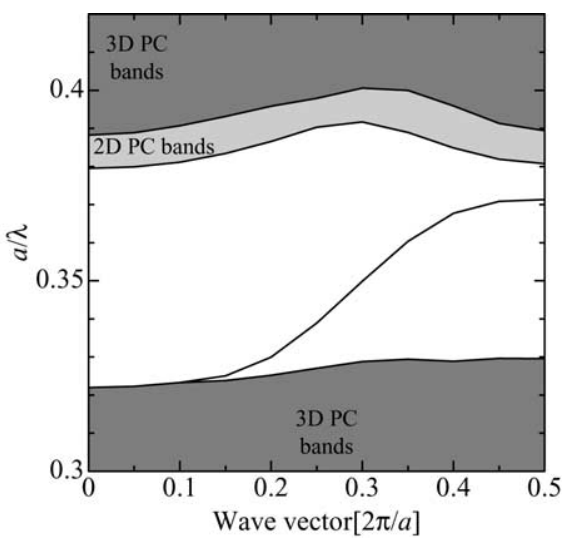

Fig. 2. Dispersion relation for an air-waveguide channel in the diamond:1 heterostructure.

inverted state by coherent resonant pumping. Such an effect is strictly forbidden in an ordinary vacuum, where the absorption and emission of light from atoms is governed by the Einstein rate equation [13]. Population inversion in conventional lasers is achieved by utilizing an additional level structure in the atom [28]. For instance, by pumping to a third atomic level that then relaxes (incoherently) to a target excited state, population inversion is achieved on the (target) second level. Here, we describe PBG architectures in which population inversion is achieved without recourse to a third level and without incoherent processes. This leads to the possibility of collective "atomic switching" [14] and the further possibility of all-optical transistor action [15] with micro-Watt power level and without the usual trade-off between switching time and switching power [29].

We briefly review the mechanism of the atomic population inversion here, the details can be found in [15] and [21]. When a coherent laser beam interacts with a two-level atom, the Hamiltonian of the system has the form $H=H_{0}+H_{\mathrm{AR}}+H_{\mathrm{AL}}$. The non-interacting Hamiltonian of the bare atomic and the photonic reservoir is $H_{0}=\hbar \Delta_{\mathrm{AL}} \sigma_{3}+\sum_{\lambda} \hbar \Delta_{\lambda} a_{\lambda}^{\dagger} a_{\lambda}$, where $\Delta_{\mathrm{AL}}=\omega_{\mathrm{A}}-\omega_{\mathrm{L}}, \Delta_{\lambda}=\omega_{\lambda}-\omega_{\mathrm{L}}, \omega_{\mathrm{A}}$ is the transition frequency of the two-level atom, $\omega_{\mathrm{L}}$ is the frequency of the laser field, and $\omega_{\lambda\}}$ the frequency of a mode $\lambda$. The interaction between atom and photonic reservoir is $H_{\mathrm{AR}}=i \hbar \sum_{\lambda} g_{\lambda}\left(a_{\lambda}^{\dagger} \sigma_{12}-\sigma_{21} a_{\lambda}\right)$ and the interaction between the atomic system and laser field is $H_{\mathrm{AL}}=\hbar \varepsilon\left(\sigma_{12}+\sigma_{21}\right)$. Here, $\sigma_{i j}=|i\rangle\langle j|(i, j=1,2)$ are the atomic excitation and de-excitation operators, and $\sigma_{3}=\sigma_{22}-\sigma_{11}$ is the atomic inversion operator. The coupling constant $\left(g_{\lambda\}}\right)$ describes the coupling between the atom and the reservoir $\lambda$-mode. The resonant Rabi frequency $\varepsilon=\boldsymbol{\mu} \cdot \boldsymbol{E} / \hbar$ describes the interaction between the atom and the laser field, where $\boldsymbol{\mu}$ is the dipole moment of the atom and $\boldsymbol{E}$ is the applied laser field amplitude.

The Hamiltonian can be simplified by introducing the dressed atomic states $\{|\tilde{1}\rangle,|\tilde{2}\rangle\}$, defined by $|\tilde{1}\rangle=$ $c|1\rangle+s|2\rangle$ and $|\tilde{2}\rangle=-s|1\rangle+c|2\rangle$, that diagonalize $H_{0}+H_{\mathrm{AL}} . \quad$ Here, $\quad c^{2} \equiv \cos ^{2}(\phi) \equiv\left[1+\operatorname{sign}\left(\Delta_{\mathrm{AL}}\right)\right.$ $\left.\left|\Delta_{\mathrm{AL}}\right| /(2 \Omega)\right] / 2$ and $s^{2} \equiv \sin ^{2}(\phi) \equiv\left[1-\operatorname{sign}\left(\Delta_{\mathrm{AL}}\right)\right.$ $\left.\left|\Delta_{\mathrm{AL}}\right| /(2 \Omega)\right] / 2$, where the generalized Rabi frequency is defined by $\Omega \equiv \sqrt{\varepsilon^{2}+\Delta_{\mathrm{AL}}^{2} / 4}$. In the dressed state basis, the total Hamiltonian becomes $H=\tilde{H}_{0}+\tilde{H}_{I}$. Here, $\tilde{H}_{0}=\sum_{\lambda} \hbar \Delta_{\lambda} a_{\lambda}^{\dagger} a_{\lambda}+\hbar \Omega \tilde{\sigma}_{3}$, and $\tilde{H}_{I}=i \hbar \sum_{\lambda} g_{\lambda}\left[a_{\lambda}^{\dagger}\left(c s \tilde{\sigma}_{3}+c^{2} \tilde{\sigma}_{12}-s^{2} \tilde{\sigma}_{21}\right)\right]+$ h.c., where $\tilde{\sigma}_{i j}=|\tilde{i}\rangle\langle\tilde{j}|(i, j=1,2)$ are the dressed atomic excitation and de-excitation operators, and $\tilde{\sigma}_{3}=\tilde{\sigma}_{22}-$ $\tilde{\sigma}_{11}$ is the dressed inversion operator. Then, we introduce the time-dependent interaction picture generated by the unitary operator $U \equiv \exp \left(-i \tilde{H}_{0} t\right)$. The interaction Hamiltonian becomes $\tilde{H}_{I}(t) \equiv U \tilde{H}_{I} U \dagger$, where

$$
\begin{aligned}
\tilde{H}_{I}(t)= & i \hbar \sum_{\lambda} g_{\lambda}\left[a _ { \lambda } ^ { \dagger } \left(c s \tilde{\sigma}_{3} \mathrm{e}^{i \Delta_{\lambda} t}+c^{2} \tilde{\sigma}_{12} \mathrm{e}^{i\left(\Delta_{\lambda}-2 \Omega\right) t}\right.\right. \\
& \left.\left.-s^{2} \tilde{\sigma}_{21} \mathrm{e}^{i\left(\Delta_{\lambda}+2 \Omega\right) t}\right)\right]+ \text { h.c. }
\end{aligned}
$$

When the laser field amplitude (described by $\hbar \varepsilon$ ) is strong enough so that the Mollow frequencies $\omega_{\mathrm{L}}$, $\omega_{\mathrm{L}}-2 \Omega$, and $\omega_{\mathrm{L}}+2 \Omega$ are pushed away from the DOS discontinuities, the Mollow spectral components at $\omega_{\mathrm{L}} \pm 2 \Omega$ experience very different density-of-states and can be described by spontaneous emission decay rates $\gamma_{ \pm}=2 \pi \sum_{\lambda} g_{\lambda}^{2} \delta\left(\omega_{\lambda}-\omega_{\mathrm{L}} \mp 2 \Omega\right)$.

The steady state solution of the expectation value of the inversion operator is (see [15] for details) $<\tilde{\sigma}_{3}>^{s t}=\left(\gamma_{-} s^{4}-\gamma_{+} c^{4}\right) /\left(\gamma_{-} s^{4}+\gamma_{+} c^{4}\right)$. For the case $\Delta_{\mathrm{AL}}>0$, coefficient $c^{2}>s^{2}$. The dressed state $|\tilde{2}\rangle$ is mostly comprised of the bare excited state $|2\rangle$. When $\gamma_{-} s^{4}>\gamma_{+} c^{4}$, the dressed state $|\tilde{2}\rangle$ is more populated in the steady state regime, which corresponds to an accumulation of atomic population on the bare excited state $|2\rangle$. In other words, at a pump intensity threshold, $\varepsilon_{\mathrm{thr}}$, defined by the condition 
$\tan ^{4} \phi=\gamma_{+} / \gamma_{-}$, the atom switches from being in an absorptive state on the lower Mollow side-band at frequency $\omega_{\mathrm{L}}-2 \Omega$ to one that provides gain to an optical pulse at the same frequency. In the absence of any LDOS jump (i.e., $\gamma_{+}=\gamma_{-}$), we recapture the atomic population behavior predicted by the Einstein rate equations. Namely, it is impossible to achieve population inversion by coherent resonant pumping. Unlike population inversion in conventional lasers, achieved by incorporating additional level structure into the atom (i.e., three- or four-level atoms), population inversion in the context of our two-level atom is achieved by incorporating a nontrivial structure into the vacuum. Moreover, the steady state atomic switching occurs through entirely coherent processes. The larger the LDOS jump (as determined by the ratio $\left.\gamma_{+} / \gamma_{-}\right)$, the lower the switching threshold.

The inversion process can also be understood by the schematic energy level of the bare atom and the dressed atom state depicted in Fig. 3. The state $|i, n\rangle$ corresponds to the state in which the atom is in its $i$ th state $(i=1,2)$ and there are $n$ photons in the background. The splitting between the states $|1, n+1\rangle$ and $|2, n\rangle$ is given by the detuning between the atomic and laser frequencies, $\Delta_{\mathrm{AL}}$. If $\Delta_{\mathrm{AL}}$ and the Rabi frequency $\varepsilon \approx\left\langle 2, n\left|H_{\mathrm{AL}}\right| 1, n+1\right\rangle / \hbar$ are small compared to $\omega_{\mathrm{L}}$, we can neglect all the coupling between different atom $\otimes$ laser manifolds. If the "atom" is in the state $|\tilde{2}, n\rangle(|\tilde{1}, n\rangle)$, it can only decay by emitting a "high" or "medium" ("medium" or "low") photon, where "high", "medium", and "low" photon have a frequency of $\omega_{\mathrm{L}}+2 \Omega, \omega_{\mathrm{L}}$, and $\omega_{\mathrm{L}}-2 \Omega$, respec-

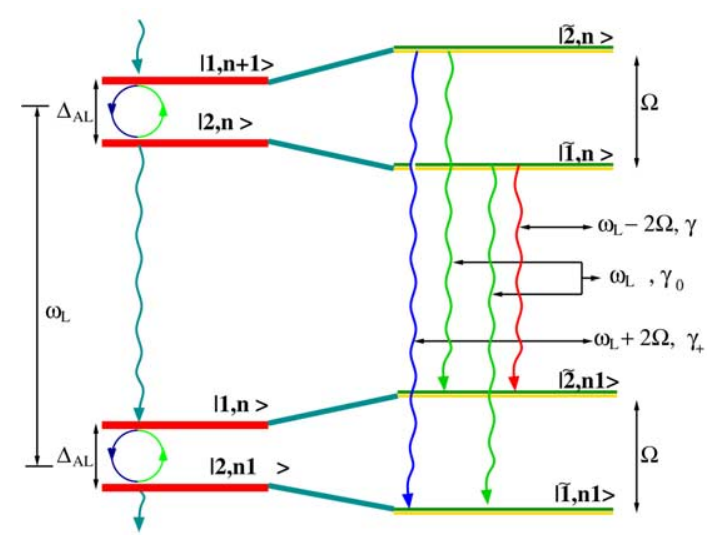

Fig. 3. Bare atom and dressed atom state diagram. tively. The decay rate of the dressed atom $\gamma_{ \pm}$is proportional to the LDOS at the corresponding photon frequency. In a steady state, the dressed atomic decay through emitting a "medium" photon will be compensated by absorbing a "medium" photon from the pumping laser. When the dressed atom experiences different decay rates at the Mollow sidebands, it will accumulate at the state associated with a slow decay rate. The bigger the LDOS jump, the faster the accumulation process. Within some parameter range, the accumulated dressed state corresponds to the excited state of the bare atom. Thus, the atomic population inversion of bare atomic states is achieved.

\section{LDOS of a single waveguide in a 3D PBG hetero-structure}

To achieve atomic population inversion with microWatt pump laser fields requires stringent conditions on the electromagnetic LDOS. In particular, a jump in the LDOS by a factor of 100 or more is required over a frequency interval that is $10^{-4}$ or less than the frequency where the LDOS jump occurs. In this section, we study the LDOS of suitable air-waveguides in a 3D PBG hetero-structure. Near the cutoff frequency of a waveguide mode, the dispersion relation can be approximated by $\omega=\omega_{\mathrm{c}}-A\left(k_{y}-k_{c}\right)^{2}$, where $k_{\mathrm{c}}$ is the wave vector corresponding to $\omega_{\mathrm{c}}$ and $A$ is a fitting constant. Consequently, the photon DOS will be $\rho_{1}(\omega)=(1 / 2) A\left(\omega_{\mathrm{c}}-\omega\right)^{-1 / 2}$, which is divergent at $\omega_{\mathrm{c}}$. However, in practice, the size of the hetero-structure (and length of the corresponding waveguide) is always finite. As a result, the LDOS divergence is replaced by a large but finite discontinuity near the cut-off frequency.

The LDOS is obtained by calculating the energy flux from a dipole inside the structure [30-32]. For concreteness, an inverse diamond:5 square spiral [23] with the parameters $[L, c, r]=[1.5,1.7,0.33]$ is used as 3D PBG cladding materials. We emphasize, however, that this serves only as a concrete illustration. The finite structure has three layers of cladding material above and below the 2D slab, and the thickness of the 2D slab $h=0.4 a$. There are five lines of rods on each side of the waveguide. The length of the waveguide is varied to determine its influence on the LDOS, for example, $7 a$ (denoted as $y 7$ ), $9 a$ (denoted as $y 9$ ), etc. 


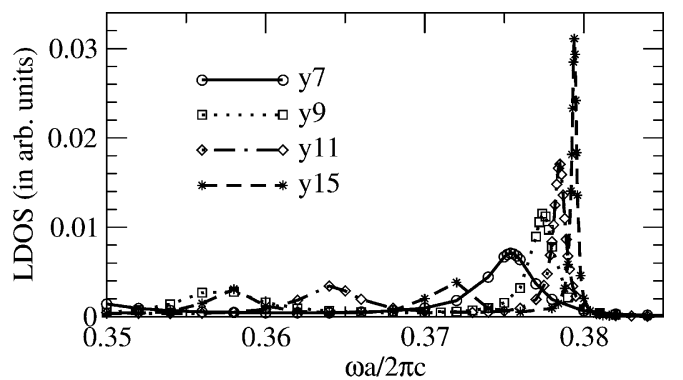

Fig. 4. The LDOS of the finite hetero-structure with different lengths in the waveguide direction. The dipole is placed at $\boldsymbol{r}=(-0.7,0.3,0) a$. The origin of the coordinate is the center of the structure.

The LDOS of the hetero-structure is shown in Fig. 4. Near the cutoff frequency $\left(\omega_{\mathrm{c}}\right)$, $\omega_{\mathrm{c}} \approx 0.38(2 \pi c / a)$, there is a peak in the LDOS that becomes more pronounced as the waveguide becomes longer. As the length of the waveguide increases, the peak value $\left(\rho_{\max }\right)$ of the LDOS increases rapidly. At the same time, the peak width decreases. When the length of the waveguide goes to infinity, the peak in the LDOS will be eventually divergent at $\omega_{\mathrm{c}}$. Below $\omega_{\mathrm{c}}$, the lowest value of the LDOS, $\rho_{\min }$, is almost the same for all the waveguides, which corresponds to the linear dispersion part of the waveguide mode. Besides the peak near $\omega_{\mathrm{c}}$, there are some smaller fringes in the LDOS when $\omega<\omega_{\mathrm{c}}$, due to finite-size effects. This is a Fabry-Perot effect arising from the photonic crystal to free space boundary at the edges of our sample [29].

We now consider in greater detail the properties of the dominant peak arising from the waveguide cutoff. If the frequency of the peak LDOS, $\rho_{\max }$, is $\omega_{\mathrm{m}}$, and the frequency at $0.5 \rho_{\max }$ (bigger than $\omega_{\mathrm{m}}$ ) is $\omega_{\mathrm{r}}$, the peak width is simply defined as $\Delta \omega=\omega_{\mathrm{r}}-\omega_{\mathrm{m}}$. For waveguides with different lengths, the peak value and peak width can be fitted to analytic functions, as shown in Fig. 5. The peak value varies as $\rho_{\max }(L) / \rho_{\min }=a_{0}+a_{1} L+a_{2} L^{2}$ and the peak width varies as $\Delta \omega(L) / \omega_{\mathrm{c}}=b \mathrm{e}^{-\alpha L}$. Here, $a_{0}, a_{1}, a_{2}, b$, and $\alpha$ are fitting parameters and $\omega_{\mathrm{c}}$ is chosen to be $0.38(2 \pi c / a)$.

Since the evaluation of the LDOS of a long waveguide by FDTD methods is very time consuming, it is useful to infer the desired properties by extrapolation. The ratio $\rho_{\max } / \rho_{\min }$ (shown in Fig. 5(a)), describes the ratio of the peak LDOS arising from a mode cutoff to the background LDOS of a linear dispersion mode.

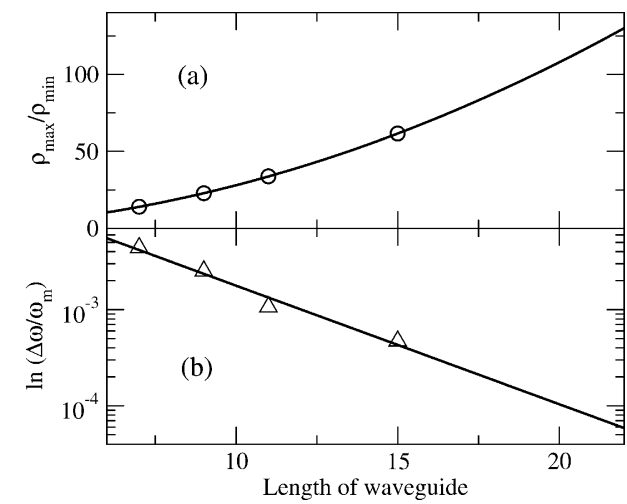

Fig. 5. Analytic functions fitted for (a) peak value and (b) peak width.

When the length of the waveguide is $20 a$, the ratio $\rho_{\max } / \rho_{\min }$ is about 100 . Meanwhile, the LDOS peak width decreases rapidly with waveguide length. In particular, the ratio $\Delta \omega / \omega_{\mathrm{c}}$ is as small as $10^{-4}$ when the waveguide length is $20 a$. Consequently, the LDOS peak of the waveguide in a 3D heterostructure is suitable to act as a "building block" in the engineering of electromagnetic LDOS.

We estimate the power of the pump laser beam passing through the waveguide to realize atomic population inversion. The Rabi frequency can be obtained by the formula $|\Omega|=2.2 \times$ $10^{8} \sqrt{I\left[\mathrm{~W} / \mathrm{cm}^{2}\right]}|\boldsymbol{d} \cdot \boldsymbol{e}| /\left(e a_{0}\right)[33]$, where $I \equiv c / 8 \pi|\boldsymbol{E}|^{2}$ is the intensity of laser beam in the unit of $W / \mathrm{cm}^{2}$, $|\boldsymbol{E}|$ is the electric field amplitude, $|\boldsymbol{d} \cdot \boldsymbol{e}|$ is the projection of the dipole moments in the electric field direction, $e$ is the electron charge, and $a_{0}$ is the Bohr radius. Usually, $|\boldsymbol{d} \cdot \boldsymbol{e}| \sim 0.1-10\left(e a_{0}\right)$. When $|\boldsymbol{d} \cdot \boldsymbol{e}|=$ $10\left(e a_{0}\right)$ and the light wavelength is $1.5 \mu \mathrm{m}, 1 \mathrm{~nm}$ shift (133 GHz shift) of a Mollow sideband from the central frequency of the Mollow spectrum requires an electric amplitude of about $10^{4} \mathrm{~V} / \mathrm{cm}$ at the location of the atom (quantum dot). If a jump within $10^{-4} \omega_{\mathrm{c}}$ is achieved in the DOS $(0.15 \mathrm{~nm}$ or $20 \mathrm{GHz}$ shift for $\lambda=1.5 \mu \mathrm{m})$, the laser electric amplitude required for atomic switching in the simple model (the single two-level atom case) of Section 2 is about $1.6 \times 10^{3} \mathrm{~V} / \mathrm{cm}$. Although the electric field amplitudes required for atomic switching are moderate, the total energy and power requirement for switching are very small, due to the submicron-scale confinement of the optical fields within a typical photonic bandgap 
waveguide geometry. For a PBG centered near 1.5 $\mu \mathrm{m}$, the required photonic crystal lattice constant $a \sim 0.4 \lambda \approx 600 \mathrm{~nm}$. One unit volume of the waveguide direction is about $5 a \times 0.3 a \times 1 a \approx 0.3$ $\mu \mathrm{m}^{3}$. The energy stored along one unit cell of the waveguide channel, at the atomic switching threshold, is about $1 \times 10^{-11} \mathrm{~nJ}$. and the required power for switching is about $1 \mu \mathrm{W}$. When a large $(\sim 1000)$ number of quantum dots responds collectively, the amplitude of the switching laser is about $5 \%$ of the holding field amplitude. In this case, the power of the modulating pump laser passing through the high group velocity (linear dispersion) waveguide mode can be $3 \mathrm{nW}$, provided that the steady holding laser intensity is just below the switching threshold.

\section{Waveguide structure for a LDOS filter}

In this section, we introduce a new micro-chip architecture that provides an LDOS filter (fork-like shape) for switching in an inhomogeneously broadened atomic distribution [29]. From the discussion in Section 3, atomic switching to a populationinverted state depends sensitively on the detuning of the atomic transition from the LDOS jump. Improperly detuned atoms in an inhomogeneously broadened ensemble will cause absorption, offsetting the gain provided by properly detuned atoms. Accordingly, we design an LDOS filter architecture, which selects correctly detuned atoms and disables incorrectly detuned atoms. We consider a $2 \mathrm{D}$ square lattice with circular rods and consider only the E-polarization mode (electric field parallel to the rods), because the field pattern in the 3D hetero-structures closely resembles the E-polarization mode in a 2D photonic crystal. The lattice constant is $a$ and the radius of rods is $r_{0}=0.2 a$, providing a full stop gap from 0.308 to $0.453(2 \pi c / a)$ for the E-polarization mode. As plotted in Fig. 6, three rows of rods are modified to create a trimodal waveguide, leading to a filter architecture of the LDOS. By completely removing two lines of rods, two air-waveguide modes are created. One (mode 3) has a lower-cutoff frequency $\left(\omega_{3}\right)$ near the center of the gap. The other (mode 2) has a linear dispersion relation near this frequency range. By reducing the radius $\left(r_{1}\right)$ of one line of rods, another waveguide mode (mode 1 ) is created with the upper-cutoff fre-

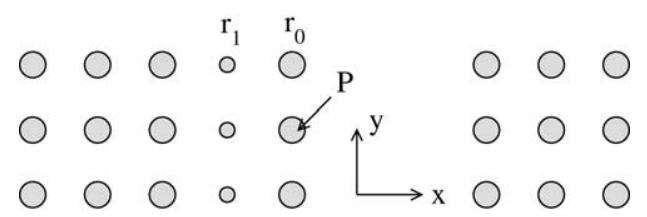

Fig. 6. Architecture for an LDOS filter for switching in an inhomogeneously broadened "atomic system." The two-level atoms or quantum dots are placed at point $\mathrm{P}$ and other equivalent points.

quency $\left(\omega_{1}\right)$ near the center of the gap. The group velocity of mode 2 near the cutoff frequencies (of modes 1 and 3 ) is $0.76 c$, where $c$ is the light velocity in a vacuum. The corresponding band structure is plotted in Fig. 7, in which $r_{0}=0.2 a$ and $r_{1}=0.116 a$.

The lower-cutoff frequency, $\omega_{3}$, of the air-waveguide (mode 3 ) is mainly determined by $r_{0}$. When $r_{0}=0.2 a, \omega_{3}$ is near the center of the gap. The uppercutoff frequency, $\omega_{1}$, of the waveguide (mode 1) can be adjusted by changing the rod radius $r_{1}$, as depicted in Fig. 8. When $r_{1}$ increases, $\omega_{3}$ is almost invariant (actually decreases very, very slowly), while the $\omega_{1}$

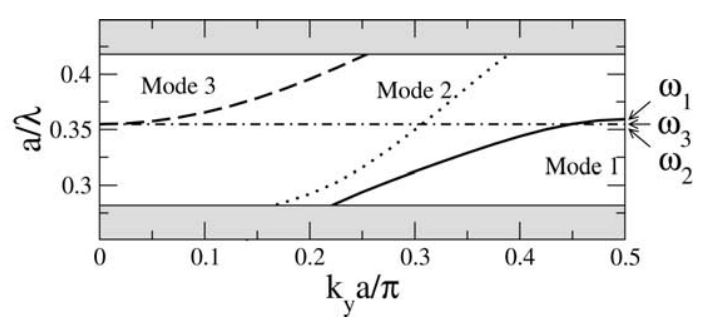

Fig. 7. The band structure of the $2 \mathrm{D}$ photonic crystal with three waveguide modes. Mode 1 (solid line) and mode 3 (dashed line) exhibit cutoffs within the PBG and contribute to sharp LDOS peaks, whereas mode 2 (dotted line) exhibits nearly linear dispersion in the same vicinity. The probe laser frequency (position of lower Mollow sideband) is indicated as $\omega_{2}$, the pump laser frequency is indicated as $\omega_{3}$, and the position of upper Mollow sideband is indicated as $\omega_{1}$.

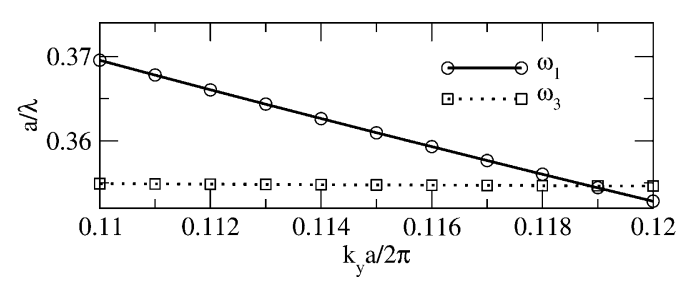

Fig. 8. The dependence of the cutoff frequencies on the radius, $r_{1}$. 


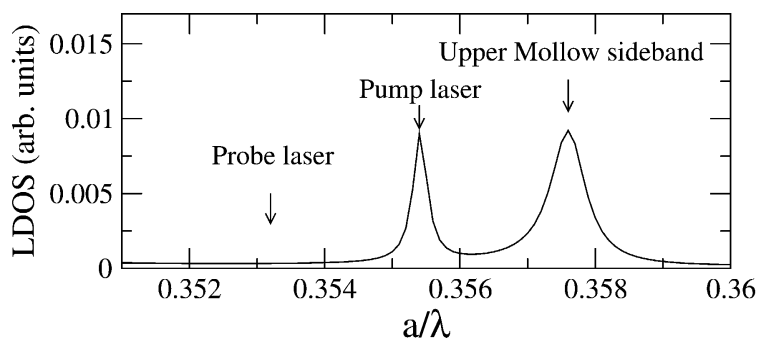

Fig. 9. The local density-of-states for $2 \mathrm{D}$ photonic crystal waveguides with the length $15 a$. The dipole is placed at the point $\mathrm{P}$ in Fig. 6.

decreases noticeably. This makes the relative position between these two cutoff frequencies adjustable by changing $r_{1}$. The waveguide (mode 2), which carries the probing laser, has a high group velocity enabling a quick response for ultrafast switching.

The LDOS of the waveguide architecture is plotted in Fig. 9. When $r_{1}=0.116 a$, the peak near $0.355(2 \pi c / a)$ corresponds to the cutoff frequency of the air-waveguide. The peak near $0.358(2 \pi c / a)$ corresponds to the cutoff frequency of the waveguide (mode 1). The steady state pumping laser frequency is adjusted to the left LDOS peak (mode 3), and the higher frequency peak (mode 1) is chosen to coincide with the upper Mollow sideband of the atomic resonances fluorescence spectrum. The probing laser is adjusted to the lower Mollow sideband. This architecture selects those atoms whose central and upper Mollow components coincide with the two LDOS peaks, while suppressing the deleterious effect of otherwise detuned atoms.

\section{Robustness of vacuum engineering to disorder}

The highly frequency selective control of spontaneous emission provided by the LDOS architectures, described above, requires high precision microfabrication of the relevant micro-chip waveguides. Here, we consider the effect of unintentional disorder on the LDOS characteristics. We first consider the W1 waveguides in a 2D PC to study the influence of random rod radius disorder. Two kinds of waveguides are studied. One is the air-waveguide, which has one line of rods removed. The other is the dielectric waveguide, which has the radius of one line of rods modified instead of removed. The 2D PC has a square lattice with circular rods, whose radius is $0.25 a$ and the dielectric constant is 11.9. The radius of the defect rods in the dielectric waveguide is $0.16 a$.

We study the case that the radius of the rod is distributed evenly, but randomly, within a range of radii. When the radius randomness is $10 \%$, i.e., the rod radius is randomly distributed between 95 and $105 \%$ of its original size $(\sim 22 \mathrm{~nm}$ diameter randomness for operation at $\lambda=1.5 \mu \mathrm{m}$ ), the LDOS of the waveguide with various random configurations is depicted in Fig. 10. For both kinds of waveguides, disorder shifts the LDOS peaks, leading to a distribution of the

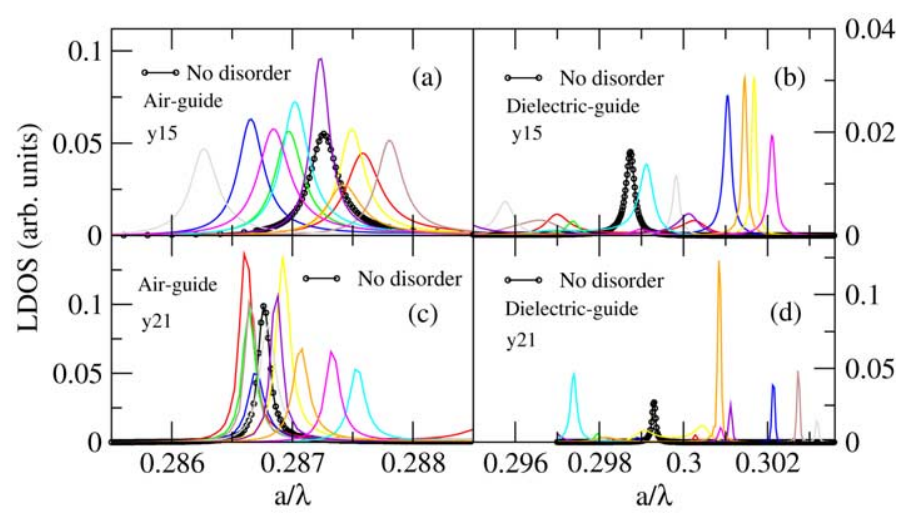

Fig. 10. The LDOS of waveguides with random radius (10\% randomness, $\sim 22 \mathrm{~nm}$ diameter randomness for rod $\left.r_{0}\right)$. The black lines with circles are the LDOS without disorder. The color lines represent various random configurations. (a) y15 air-waveguide; (b) y15 dielectric waveguide; (c) y21 air-waveguide; and (d) y21 dielectric waveguide. Length of the waveguide is $15 a$ for (a) and (b), $21 a$ for (c) and (d), respectively. 


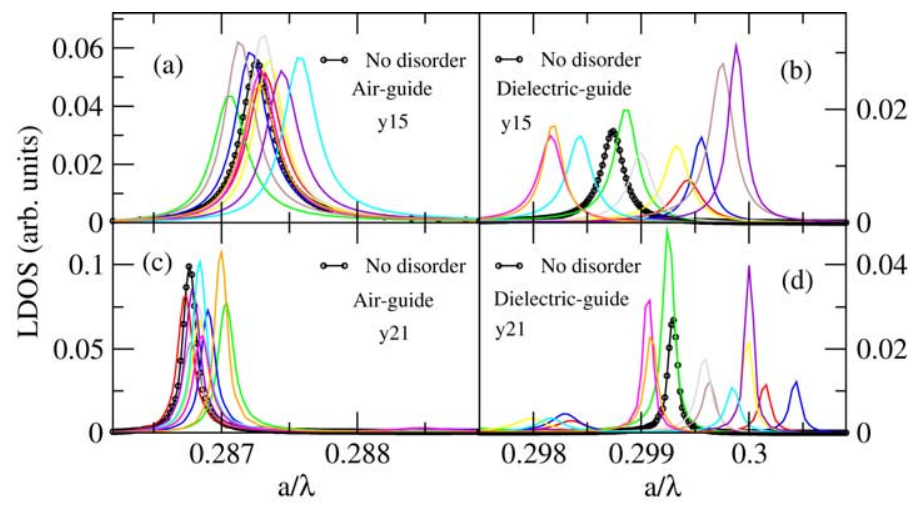

Fig. 11. The LDOS of waveguides with random radius ( $4 \%$ randomness, $\sim 9 \mathrm{~nm}$ diameter randomness for rod $r_{0}$ ). The black lines with circles are the LDOS without disorder. The color lines represent various random configurations. (a) y15 air-waveguide; (b) y15 dielectric waveguide; (c) y21 air-waveguide; and (d) y21 dielectric waveguide. Length of the waveguide is $15 a$ for (a) and (b), $21 a$ for (c) and (d), respectively.

peak positions for various randomness configurations. The shape of the LDOS peak remains the same for airwaveguides, but the height of the peak fluctuates. For the dielectric waveguides, the LDOS peak becomes very broad in a few cases. The height of the peak also changes substantially for the dielectric waveguides. air-waveguides are more robust to disorder than dielectric waveguides.

The distribution range of the LDOS peak is almost the same for the waveguide of $15 a$ and $21 a$ in length. However, the height of the LDOS changes more in longer waveguides than in shorter waveguides. Moreover, dielectric waveguides are more vulnerable than air-waveguides in regard to LDOS height variations.

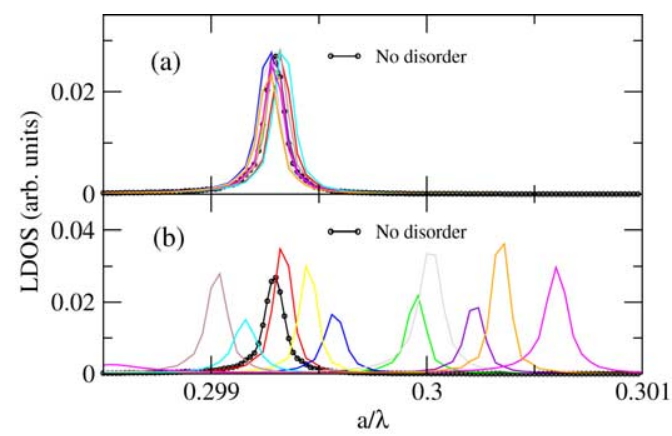

Fig. 12. The LDOS of dielectric waveguides (21 $a$ in length) with random radius ( $4 \%$ randomness). The black lines with circles are the LDOS without disorder. The color lines represent various random configurations. (a) Only rods outside the defect line have a random distribution. (b) Only rods in the defect line have a random distribution.
When the radius randomness is $4 \%(\sim 9 \mathrm{~nm}$ diameter randomness for a waveguide operating at $\lambda=1.5 \mu \mathrm{m}$ ), the influence of disorder is greatly reduced for both kinds of waveguides (see Fig. 11). The LDOS peak shapes are preserved in almost all random configurations for the dielectric waveguides, even for waveguides with $21 a$ in length. To determine which dielectric rods contribute most to LDOS fluctuations, two random cases are studied further. In the first case, all rods have a random radius, except the rods in the defect line (Fig. 12a). In the second case, only the rods in the defect line have a random radius (Fig. 12b). Clearly, disorder of the rods in the defect line plays a dominant role. This suggests that within the "active region" (the defect line), precise control of the fabrication process (nanometer scale resolution) may prove advantageous. Outside this limited active region, the PBG micro-chip can sustain substantial disorder without degradation of functionality.

A more delicate question arises in the case of the multimode LDOS filter architecture described in Fig. 6. Here, disorder can, in principle, cause scattering between modes, thereby smearing out the sharp LDOS features and the associated filter function. Below, we study the effect of rod radius randomness on the trimodal waveguide used for switching in an inhomogeneously broadened collection of quantum dots. In Fig. 13(a) and (c), where the radius randomness is $4 \%$, no mode mixing can be found, when the original (with no disorder) LDOS peak separation at $1.5 \mu \mathrm{m}$ wavelength is $\sim 4 \mathrm{~nm}$. The system behaves like two independent 


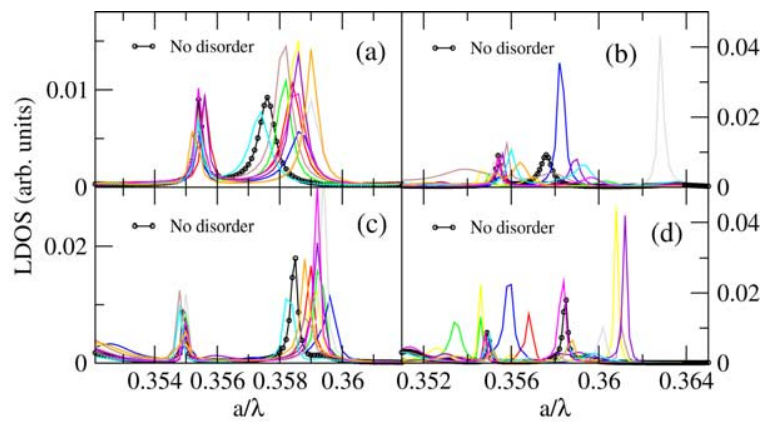

Fig. 13. The LDOS of three mode waveguides with random radius: (a) $4 \%$ randomness, $15 a$ long; (b) 10\% randomness, $15 a$ long; (c) $4 \%$ randomness, $21 a$ long; and (d) $10 \%$ randomness, $21 a$ long. All figures have 10 random configurations and the corresponding LDOS are plotted by color lines.

waveguides, and the LDOS is just a simple sum of two waveguides with radius disorder. Increasing the waveguide length only increases the LDOS height variations caused by the randomness. In Fig. 13(b) and (d), with $10 \%$ radius randomness, mode mixing is weak. However, the variation in the LDOS peak position (with disorder) exceeds the original peak separation (in the case of no disorder). This would lead to an LDOS filter device whose operating characteristics lack sufficient predictability. To compensate this unpredictability, it may be useful to introduce some refractive index tuning mechanism to "re-configure" the device to operate with prescribed characteristics [34].

We estimate that in order to achieve well-defined LDOS peaks separated by $1 \mathrm{~nm}$, the diameter randomness should be no more than $4 \mathrm{~nm}$. This estimation is based upon the apparent (approximate) linear dependence of the LDOS peak position variance on the degree of rod randomness. An analogous linear frequency shift with the rod radius (in the absence of disorder) is seen in Fig. 8. A detailed and precise relationship between the rod randomness distribution and the LDOS peak position distribution requires further study. For collective atomic switching to occur for low power pump fields, a small peak separation is desirable [29].

\section{Conclusion}

In summary, we have illustrated the utility of electromagnetic vacuum engineering for coherent all-optical atomic switching. Other illustrations of sculpting the vacuum include a novel one-atom laser with engineered photon output statistics [22]. This suggests that a number of quantum electrodynamic model Hamiltonians, leading to novel quantum effects, can be realized within a PBG micro-chip by careful design and synthesis of dielectric microstructure. Waveguides and cavities in a hetero-structure consisting of a 2D photonic crystal micro-chip embedded in a 3D PBG material are used as "building blocks" to create LDOS peaks inside the PBG. Unlike commonly studied high-Q optical microcavity-based LDOS peaks, this waveguide based LDOS structure can co-exist with high group velocity waveguide modes for high speed data processing and transfer. The fundamental challenge is one of ultra-high precision microfabrication in the immediate vicinity of the active region of device architectures. While the background PBG cladding structure can accommodate substantial disorder without compromising device functionality, nearly nanometer scale structure precision may be desirable for a small number of dielectric rods in the core of the active device. This architectural precision may be a requirement for ultra-low-threshold all-optical switching and transistor functionality.

\section{Acknowledgment}

This work was supported by the Natural Sciences and Engineering Research Council of Canada.

\section{References}

[1] S. John, Phys. Rev. Lett. 58 (1987) 2486.

[2] E. Yablonovitch, Phys. Rev. Lett. 58 (1987) 2059.

[3] H. Kosaka, T. Kawashima, A. Tomita, M. Notomi, T. Tamamura, T. Sato, S. Kawakami, Phys. Rev. B 58 (1998) R10096R10099.

[4] A. Chutinan, S. John, O. Toader, Phys. Rev. Lett. 90 (2003) 123901.

[5] V. Bykov, Sov. J. Quant. Electron. 4 (1975) 861.

[6] J.G. Fleming, S.Y. Lin, I. El-Kady, R. Biswas, K.M. Ho, Nature (London) 417 (2002) 52

[7] T. Quang, M. Woldeyohannes, S. John, G.S. Agarwal, Phys. Rev. Lett. 79 (1997) 5238.

[8] P. Russell, Science 299 (2003) 358.

[9] A. Chutinan, S. John, submitted for publication.

[10] E.P. Petrov, V.N. Bogomolov, I.I. Kalosha, S.V. Gaponenko, Phys. Rev. Lett. 81 (1998) 77. 
[11] S. John, J. Wang, Phys, Rev. B 43 (1991) 12772.

[12] M. Florescu, S. John, Phys. Rev. A 64 (2001) 033801.

[13] R. Loudon, The Quantum Theory of Light, Oxford University Press, Oxford, 2000.

[14] S. John, T. Quang, Phys. Rev. Lett. 78 (1997) 1888.

[15] S. John, M. Florescu, J. Opt. A: Pure Appl. Opt. 3 (2001) S103.

[16] H.M. Gibbs, Optical Bistability: Controlling Light with Light, Academic Press, New York, 1985.

[17] H.M. Gibbs, S.L. McCall, T.N.C. Venkatesan, Phys. Rev. Lett. 36 (1976) 1135.

[18] P.St. Russell, Electron. Lett. 29 (1993) 1228.

[19] D.A.B. Miller, Optical computing, in: B.S. Wherrett, F.A.P. Tooley (Eds.), in: Proceedings of the Thirty-fourth Scottish Universities Summer School in Physics, Heriot-Watt University, Edinburgh, Scotland, 1989.

[20] J. Lefebvre, P.J. Poole, J. Fraser, G.C. Aers, D. Chithrani, R.L. Williams, Heriot-Watt University, Edinburgh, Scotland, J. Cryst. Growth 2342002391.

[21] M. Florescu, S. John, Phys. Rev. A 69 (2004) 053810.

[22] L. Florescu, S. John, T. Quan, R. Wang, Phys. Rev. A 69 (2004) 013816.
[23] O. Toader, S. John, Phys. Rev. E 66 (2002) 016610.

[24] S. Noda, K. Tomoda, N. Yamamoto, A. Chutinan, Science 289 (2000) 604.

[25] O. Toader, M. Berciu, S. John, Phys. Rev. Lett. 90 (2003) 233901.

[26] A. Blanco, E. Chomski, S. Grabtchak, M. Ibisate, S. John, S.W. Leonard, C. Lopez, F. Meswguer, H. Miguez, J.P. Mondia, G.A. Ozin, O. Toader, H.M. van Driel, Nature (London) 405 (2000) 437.

[27] S.R. Kennedy, M.J. Brett, M. Hernan, T. Ovidiu, S. John, Photonics Nanostruct.: Fundam. Appl. 1 (2003) 37.

[28] P.W. Milonni, J.H. Eberly, Lasers, Wiley, New York, 1988.

[29] R. Wang, S. John, submitted for publication.

[30] T. Suzuki, P.K.L. Yu, J. Opt. Soc. Am. B 12 (1995) 570.

[31] K. Sakoda, Optical Properties of Photonic Crystals, Springer, Berlin, 2001.

[32] C. Hermann, O. Hess, J. Opt. Soc. Am. B 19 (2002) 3013.

[33] B.W. Shore, The Theory of Coherent Atomic Excitation, Wiley, New York, 1990.

[34] K. Busch, S. John, Phys. Rev. Lett. 83 (1999) 2646. 